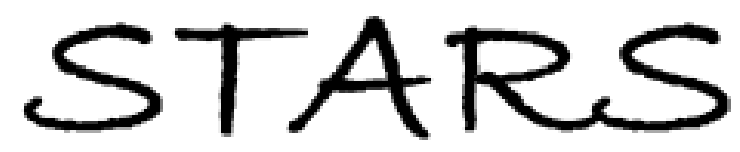

University of Central Florida

STARS

Faculty Bibliography 2000s

Faculty Bibliography

$1-1-2003$

\title{
Laser synthesis of carbon-rich SiC nanoribbons
}

I. A. Salama

University of Central Florida

N. R. Quick

A. Kar

University of Central Florida

Find similar works at: https://stars.library.ucf.edu/facultybib2000

University of Central Florida Libraries http://library.ucf.edu

This Article is brought to you for free and open access by the Faculty Bibliography at STARS. It has been accepted for inclusion in Faculty Bibliography 2000s by an authorized administrator of STARS. For more information, please contactSTARS@ucf.edu.

\section{Recommended Citation}

Salama, I. A.; Quick, N. R.; and Kar, A., "Laser synthesis of carbon-rich SiC nanoribbons" (2003). Faculty Bibliography 2000s. 2568.

https://stars.library.ucf.edu/facultybib2000/2568

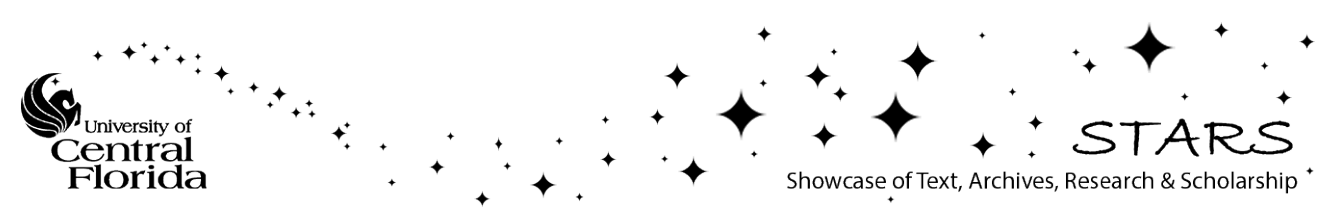




\section{Laser synthesis of carbon-rich SiC nanoribbons}

Cite as: Journal of Applied Physics 93, 9275 (2003); https://doi.org/10.1063/1.1570928

Submitted: 25 October 2002 . Accepted: 10 March 2003 . Published Online: 19 May 2003

I. A. Salama, N. R. Quick, and A. Kar

\section{ARTICLES YOU MAY BE INTERESTED IN}

Electronic structures of SiC nanoribbons

The Journal of Chemical Physics 129, 174114 (2008); https://doi.org/10.1063/1.3006431

Defect structure of carbon rich $a-\mathrm{SiC}: \mathrm{H}$ films and the influence of gas and heat treatments Journal of Applied Physics 84, 786 (1998); https://doi.org/10.1063/1.368138

Characterization of excess carbon in cubic SiC films by infrared absorption Journal of Applied Physics 85, 3377 (1999); https://doi.org/10.1063/1.369686

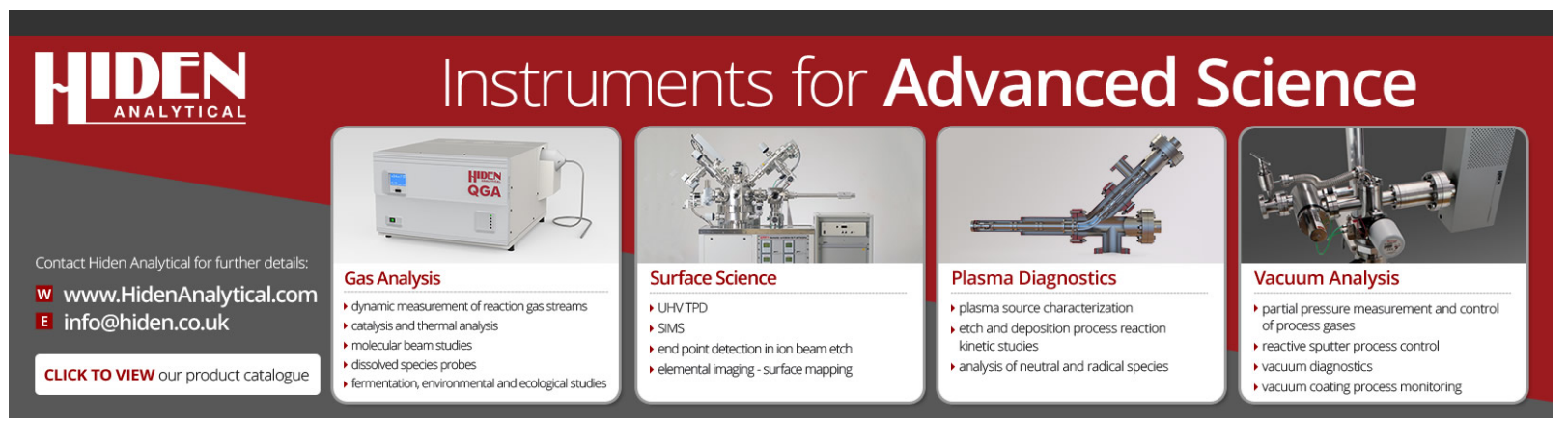




\title{
Laser synthesis of carbon-rich SiC nanoribbons
}

\author{
I. A. Salama \\ Laser-Aided Manufacturing, Materials and Micro-Processing Laboratory (LAMMMP), School of Optics, \\ Mechanical, Materials, and Aerospace Engineering Department, Center for Research and Education \\ in Optics and Lasers (CREOL), University of Central Florida, Orlando, Florida 32816-2700 \\ N. R. Quick \\ Applicote Associates, 894 Silverado Court, Lake Mary, Florida 32746 \\ A. $\mathrm{Kar}^{\mathrm{a})}$ \\ Laser-Aided Manufacturing, Materials and Micro-Processing Laboratory (LAMMMP), School of Optics, \\ Mechanical, Materials, and Aerospace Engineering Department, Center for Research and Education \\ in Optics and Laser (CREOL), University of Central Florida, Orlando, Florida 32816-2700
}

(Received 25 October 2002; accepted 10 March 2003)

\begin{abstract}
A nanosecond pulsed laser direct-write and doping (LDWD) technique is used for the fabrication of carbon-rich silicon carbide nanoribbons heterostructure in a single crystal $4 \mathrm{H}-\mathrm{SiC}$ wafer. Characterization by high-resolution transmission electron microscope and selected area electron diffraction pattern revealed the presence of nanosize crystalline ribbons with hexagonal graphite structure in the heat-affected zone below the decomposition temperature isotherm in the $\mathrm{SiC}$ epilayer. The nanoribbons exist in three layers each being approximately 50-60 nm thick, containing 15-17 individual sheets. The layers are self-aligned on the (0001) plane of the SiC epilayer with their $c$ axis at $87^{\circ}$ to the incident laser beam. The LDWD technique permits synthesis of heterostructured nanoribbons in a single step without additional material or catalyst, and effectively eliminates the need for nanostructure handling and transferring processes. (C) 2003 American Institute of Physics. [DOI: 10.1063/1.1570928]
\end{abstract}

\section{INTRODUCTION}

Recently, there has been a growing expectation that nanostructures such as carbon nanowires $(\mathrm{CNW})$, carbon nanotubes (CNT), and carbon nanoribbons (CNR) of different materials will have a great impact on the electronic device technology. These different nanostructures exhibit attractive mechanical, chemical, and electronic properties such as the electronic emitting capabilities and adjustable electric conductivity. ${ }^{1-4}$ Particular interest is drawn toward using the nanoribbons for interconnect integration and nanosensor applications. ${ }^{5,6}$ Fabrication of a heterostructure consisting of a wide band-gap semiconductor and a carbon nanostructure (CNT, CNW, or CNR) is of particular interest, since it combines the advantages of both nanosize configuration and wide band-gap semiconductors. Such nanoscale heterostructures are expected to promote new device applications, and enhance the performance of existing devices, with a great impact on the microelectronics technology. ${ }^{2}$

Among wide band-gap semiconductor materials, silicon carbide $(\mathrm{SiC})$ is by far the most mature technology for hightemperature and high power electronic applications. Due to the large band gap $\left(E_{g}=3.0\right.$ to $\left.3.5 \mathrm{eV}\right)$ of $\mathrm{SiC}$, it supports a very high breakdown field (3-5 MV/cm), typically six times higher than $\mathrm{Si}$, and permits low losses. In the hightemperature and/or high-frequency regimes where $\mathrm{SiC}$ devices are typically expected to operate, carrier mobility is

a) Author to whom correspondence should be addressed; electronic mail: akar@creol.ucf.edu sufficiently high. The outstanding thermal conductivity of $\mathrm{SiC}\left(350-490 \mathrm{~W} \mathrm{~m}^{-1} \mathrm{~K}^{-1}\right)$ - three times higher than silicon and seven times higher than $\mathrm{GaAs}$ - allows $\mathrm{SiC}$ to remove heat more efficiently causing lower junction temperatures and therefore the cooling hardware is reduced for $\mathrm{SiC}$ devices. ${ }^{\text {? }}$

The fabrication of CNT-carbide heterostructure was reported using catalytic vapor growth, vapor-solid, and direct solid-solid reactions. Dia et $a l .{ }^{8}$ demonstrated the synthesis of carbide nanorods from multiwall CNTs (MWCNTs) by a catalytic vapor growth method. The major drawback for this process is the difficulty of controlling the reaction region along the nanotube which generates a compositional variation at the interface making it difficult to identify the carbide-nanotube junction. Later, Hu et al. ${ }^{9}$ fabricated a heterostructure of Si nanowires and MWCNT using the same method. Typical rectifying contact between the CNT and $\mathrm{Si}$ nanowires was obtained, but the lack of process control leading to $\mathrm{Si}$ amorphization, oxidation, catalyst contamination, and segregation at the junction interface limited the scope of the catalytic vapor growth technique as a viable method for nanoscale heterostructure fabrication. To produce a carbide/ CNT nanoheterostructure, Zhang $e t$ al. $^{2}$ transferred prefabricated single wall CNTs (SWCNTs) to different substrates $(\mathrm{M})$, where $\mathrm{M}$ can be either a transition metal (Ti and $\mathrm{Nb}$ ) or a semimetal $(\mathrm{Si})$ substrate. Then, they transformed these (SWCNT) bundles to the corresponding metal carbide via a solid-solid reaction, i.e., $\mathrm{C}$ (nanotubes) $+\mathrm{M}$ ( solid) $\rightarrow \mathrm{MC}$ (solid). The requirements for the solid-solid reaction are an ultrahigh vacuum, to avoid the formation of any volatile re- 
actant, and high temperature. The continuous transformation of the SWCNT to the metal carbide is controlled by the diffusion of the metal (M) to the nanotube surface and then through the thin carbide layer at the interface. This requires a continued elevation of the ambient temperature to maintain sufficient diffusivity of $\mathrm{M}$ into the binary carbide. Using this technique, Zhang et $a .^{2}$ demonstrated the ability to weld an individual bundle of SWCNT to a Ti-made scanning tunneling microscope tip forming a robust nanoheterostructure. The resulting junction showed an ohmic current-voltage characteristic that improves by further annealing at high temperature. The solid-solid reaction method is more controllable than the vapor-solid reaction and seems to produce a more defined interface at the nanojunction. However, the prerequisite of manipulating, transferring, and aligning the SWCNT in partial contact with the metal substrate at room temperature and the difficulty of keeping this structure aligned at high temperature is practically a serious drawback.

The approach to fabricate in situ a carbon nanostructure (CNT, CNR, and CNW) in a carbide substrate with a defined interface is a solution to the nanomanipulation problem with an attendant reduction in the fabrication steps. Specific to the $\mathrm{SiC}$ material is the sublimation decomposition that occurs upon heating in a controlled ambient at high temperature $\left(\geqslant 1600^{\circ} \mathrm{C}\right.$ ) leading to a selective $\mathrm{Si}$ desorption at the $\mathrm{SiC}$ surface. This was utilized by various researchers ${ }^{1,10,11}$ to produce a carbon nanostructure at the surface of different $\mathrm{SiC}$ polytypes. The resulted nanostructure was observed to possess strong orientation dependence on the polytype, the crystallographic orientation, and the polarity of the $\mathrm{SiC}$ surface. For instance, Kusunoki et al. ${ }^{10}$ reported the epitaxial formation of graphite monolayer on the (0001)-Si face of a $6 \mathrm{H}-$ $\mathrm{SiC}$ single crystal while on the (0001)-C face, graphite particles, and carbon nanocaps are formed particularly at the sharp surface steps of the decomposed SiC.

In this article, we present the laser direct-write ${ }^{12,13}$ synthesis of a carbon-rich $\mathrm{SiC}$ nanoribbons heterostructure in a single crystal $4 \mathrm{H}-\mathrm{SiC}$ substrate. The laser processing parameters such as the laser energy density, spot size, scanning speed, and ambient gas govern the formation of isotherms and the shape and size of the interisotherm regions in the substrate. These isotherms influence the formation of the nanoribbons via selective evaporation of $\mathrm{Si}, \mathrm{SiC}$ decomposition, and solid-state diffusion.

\section{EXPERIMENT}

Single crystal $4 \mathrm{H}-\mathrm{SiC}$ wafer with polished (0001)-Si face is used in this study for fabricating the nanoribbons $/ \mathrm{SiC}$ structure. The wafer has a low-doped $\left(5 \times 10^{15} \mathrm{~cm}^{-3}\right.$ $\pm 25 \%), n$-type, $10 \mu \mathrm{m}( \pm 10 \%)$ thick epilayer grown onaxis $\left[(0001) \pm 0.25^{\circ}\right]$ over a $50.8 \mathrm{~mm}$ diameter $4 \mathrm{H}-\mathrm{SiC}$ substrate.

To fabricate the $\mathrm{C}$-rich $\mathrm{SiC}$ nanoribbon, laser irradiation experiments were conducted using a $Q$-switched Nd:YAG laser of wavelength $1064 \mathrm{~nm} .1 \mathrm{~mm}$ wide and $7 \mathrm{~mm}$ long region was irradiated with laser pulses of repetition rate $f$ $=35 \mathrm{kHz}$, pulse duration (on-time) $\tau_{P}=260 \mathrm{~ns}$, pulse energy $E_{P}=0.6 \mathrm{~mJ} / \mathrm{pulse}$, laser beam diameter $D=1 \mathrm{~mm}$, and laser scanning speed $V=5 \mathrm{~mm} / \mathrm{s}$. This region was irradiated three times in a nitrogen ambient $(1 \mathrm{~atm})$ before taking the sample out of the processing chamber. The incident fluence of a single pulse, which is given by $\phi_{P}=E_{p} / A_{i}$, where $A_{i}$ is the irradiated area given by $A_{i}=\left(\pi d^{2} / 4\right)+D V \tau_{P}$ for a circular spot, is found to be $76.4 \mathrm{~mJ} / \mathrm{cm}^{2}$ and the pulse intensity, which is given by $I_{p}=\left(\phi_{P} / \tau_{P}\right)$, is $293.3 \mathrm{~kW} / \mathrm{cm}^{2}$. However, a fraction of this energy is absorbed by the workpiece because the transmittance and reflectance of the $4 \mathrm{H}-\mathrm{SiC}$ sample used in this study were measured to be 0.4 and 0.15 , respectively, at $1064 \mathrm{~nm}$ wavelength. ${ }^{14}$ Focused ion beam (FIB) milling was used to prepare $5 \mu \mathrm{m} \times 8 \mu \mathrm{m} \times 1200 \AA$ section of the laser-irradiated $4 \mathrm{H}-\mathrm{SiC}$ wafer for microscopic studies. High-resolution transmission electron microscope (HRTEM), Techni F 30, equipped with a windowless Link energy dispersive spectroscopy (EDS) analyzer was used to study the laser-fabricated nanostructure and to obtain selected area electron diffraction (SAED) patterns for different regions in the laser-treated wafer.

\section{RESULTS}

Scanning electron microscope examination of the laserirradiated $\mathrm{SiC}$ wafer indicated the formation of a U-shaped trench of $5 \mu \mathrm{m}$ depth, following the scanned laser beam at the sample surface. ${ }^{14} \mathrm{~A}$ transmission electron microscope (TEM) micrograph of the graded structure formed in the laser-treated region (cross section of the U-shaped trench) is shown in Fig. 1(a). Three distinct regions are observed in this micrograph below the Pt layer deposited during the sample preparation by the FIB milling. The top region contains graphite sheets that lie just below the free surface at the bottom of the trench, and extend longitudinally over the entire laser scanning length and transversely over a small distance. The middle region is a carbon-rich $\mathrm{SiC}$ layer, and the bottom region contains the nanoribbons. These three regions are confined within a small depth $(1.1 \mu \mathrm{m})$ in the original $\mathrm{SiC}$ epilayer. In this study, the nanoribbon region was found to contain three carbon-rich nanoribbons with Si-rich strips interleaving the ribbons. It was also observed that the width of the nanoribbon region decreases symmetrically in the transverse direction [Fig. 1(b)].

A schematic representation of the laser irradiation process is presented in Fig. 2 showing the incident laser beam, laser scanning direction, and the dimensions of various regions produced by the laser treatment. In Fig. 2, AA*, A*E, and $\mathrm{EG}^{*}$ are the graphite, $\mathrm{C}$-rich and nanoribbon regions, respectively. In the carbon-rich region $\left(\mathrm{A}^{*} \mathrm{E}\right), \mathrm{Si} / \mathrm{C}$ atomic ratio increases from 0.0 at $\mathrm{A}^{*}$ (the bottom of the graphite sheets) to $\sim 0.36$ at $\mathrm{E}$ (the top of the nanoribbon region). Below the level E, the atomic distribution of both silicon and carbon shows an alternating pattern [Fig. 2(b)] whose morphology is observed to be alternately aligned nanoribbons and Si-rich strips. The ribbons have higher carbon content (85-90 at. \% C, $10-15$ at. \% Si) than the strips (75-77 at. \% C, $22-25$ at. \% Si). This compositional variation abruptly stops at the lower boundary of the last ribbon (level $\mathrm{G}^{*}$ in Fig. 2) below which the $\mathrm{SiC}$ epilayer maintains its original stoichiometry. 

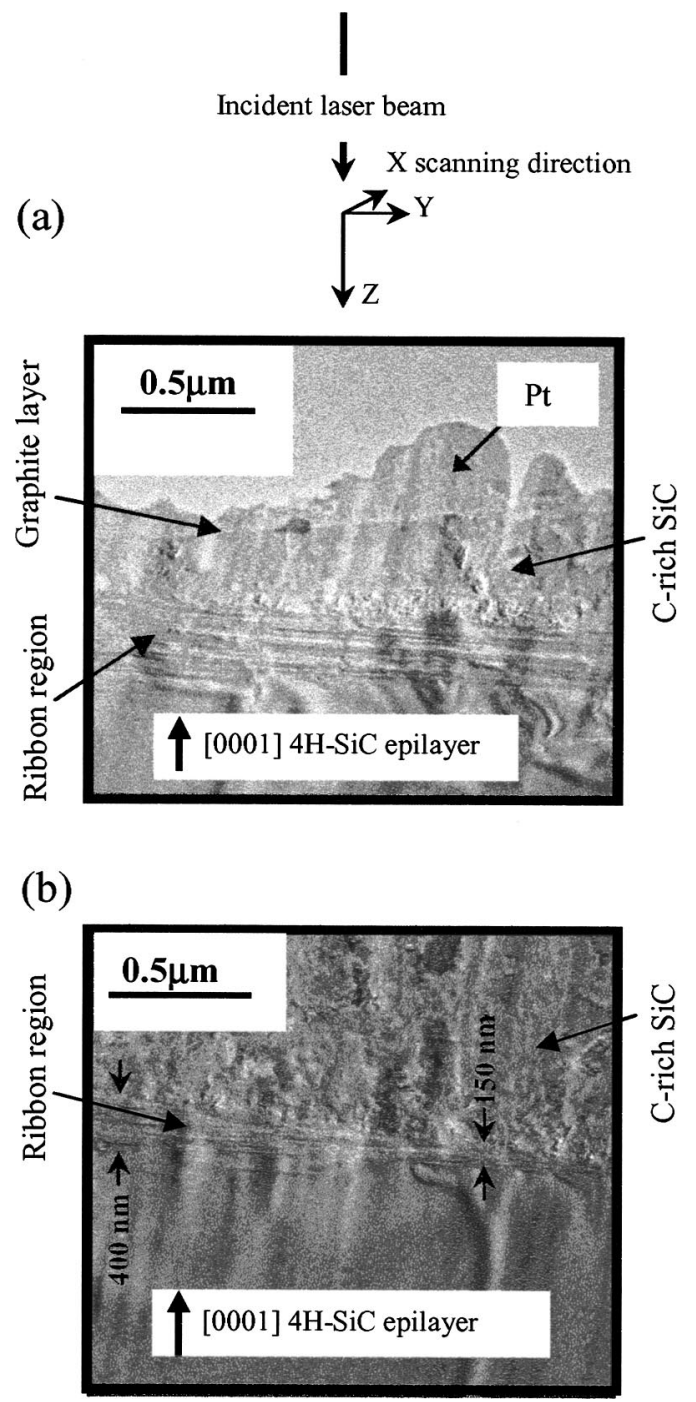

FIG. 1. TEM cross section of the laser-irradiated SiC wafer showing (a) the different regions formed after laser treatment: Platinum $(\mathrm{Pt})$ layer deposited during the TEM sample preparation by FIB, graphite sheets, carbon-rich $\mathrm{SiC}$ phase, ribbon region, and the original $\mathrm{SiC}$ epilayer, and (b) the variation in the thickness of the ribbon region. The maximum thickness $(500 \mathrm{~nm})$ occurs at the laser beam center. The TEM image in (b) was taken at $6 \mu \mathrm{m}$ away from the laser beam center.

A HRTEM lattice image of the graphite layer [region $\mathrm{AA}^{*}$ in Fig. 2(a)] containing approximately nine to ten graphite sheets is shown in Fig. 3. The thickness of the layer is $3.37 \mathrm{~nm}$. Figure 3 also shows sharp lattice fringes with an interlayer distance of $0.324 \mathrm{~nm}$ which is close to the intersheet spacing of the hexagonal graphite structure. ${ }^{15}$ Analysis of SAED pattern, inset in Fig. 3, indicated that the sheets are grown parallel to the (0001)-Si face of the $\mathrm{SiC}$ epilayer. These electron diffraction patterns were obtained with an electron beam incident parallel to the [1120] direction of the $\mathrm{SiC}$ epilayer. The amorphous contrast in the upper left-hand side corner of the image in Fig. 3 is attributed to the platinum layer deposited on the cross-sectional (TEM) sample during the FIB milling process.

Figure 4 is a TEM image of an individual nanoribbon [GG* in Fig. 2(b)] below which the original SiC epilayer remains unchanged during the laser treatment. Each nanorib- (a)

$\downarrow$

Incident laser beam

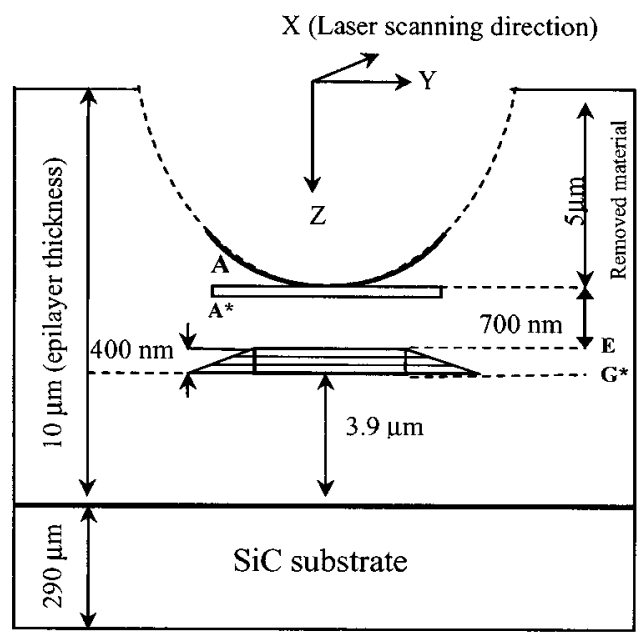

(b)

Si-rich strips

( $\mathrm{Si}=25-30$ at. $\%, \mathrm{C}=70-75$ at. $\%)$

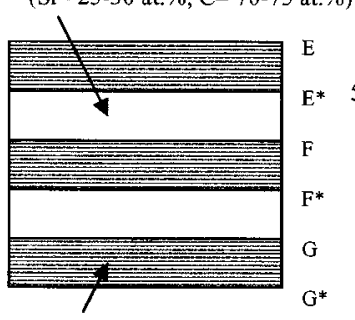

(c)

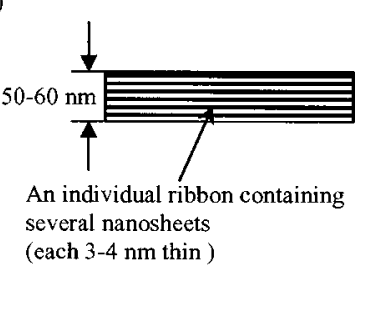

Carbon-rich ribbon

( $\mathrm{Si}=10$ at. $\%, \mathrm{C}=90 \mathrm{at} . \%)$

FIG. 2. (a) Schematic diagram of different regions (not to scale) shown in Fig. 1 in the entire epilayer of the $4 \mathrm{H}-\mathrm{SiC}$ wafer, (b) nanoribbon region and (c) a single ribbon.

bon (e.g., GG*) consists of 17-20 nanosheets that are aligned parallel to the ribbon boundaries. Figure $4(\mathrm{~b})$ is a SAED pattern of the nanoribbon $\mathrm{GG}^{*}$, indicating the presence of an additional spot diffraction pattern superimposed

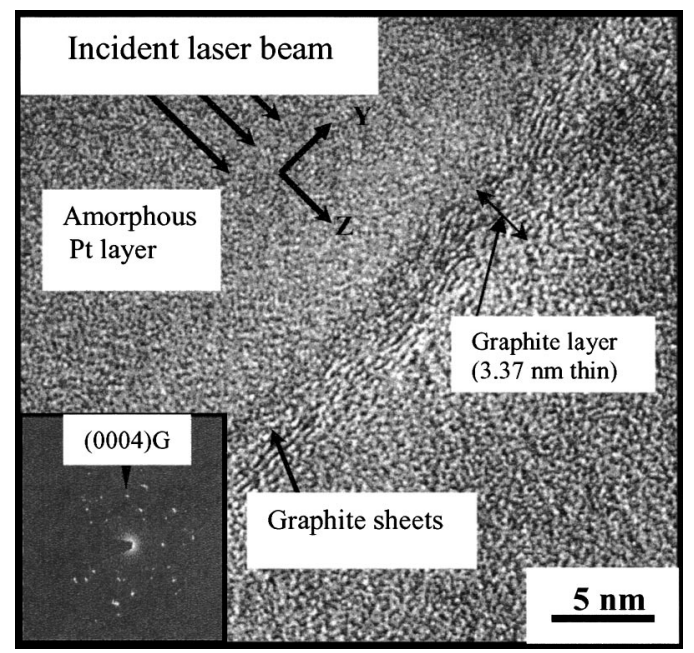

FIG. 3. HRTEM image of the graphite layer showing graphite sheets formed in the top region of the laser-irradiated $\mathrm{SiC}$ wafer. The inset is the SAED pattern of the graphite sheets indicating the hexagonal graphite structure of the sheets. The laser beam scanning direction is along the $X$ axis. 
(a)

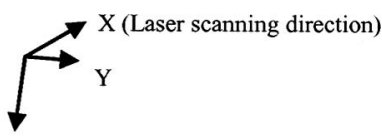

Z- Incident laser beam

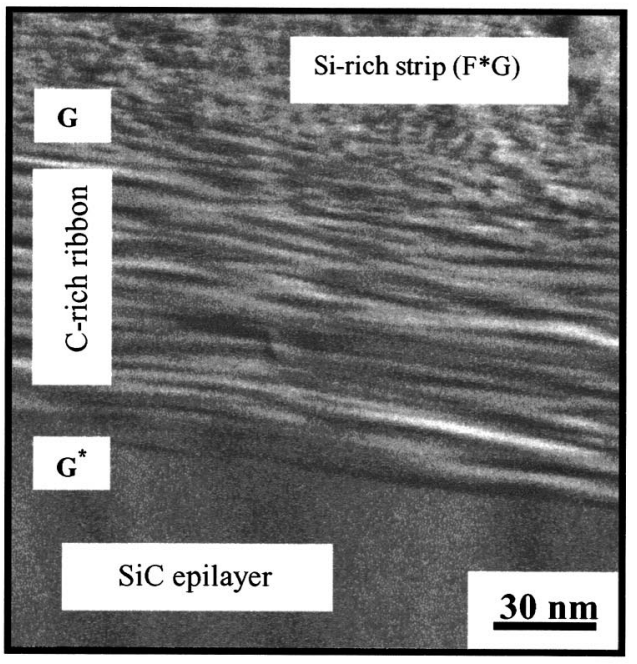

(b)

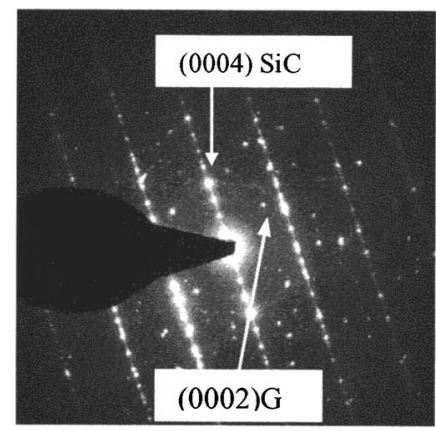

(c)

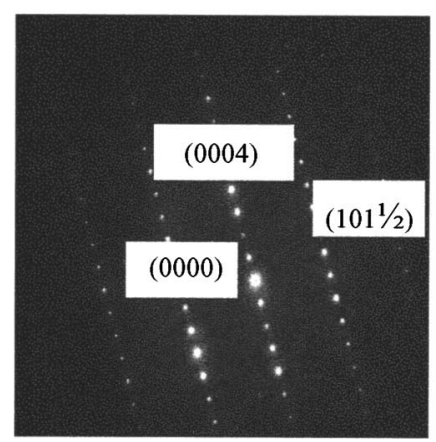

FIG. 4. (a) HRTEM image of a single carbon-rich ribbon [ribbon $\mathrm{GG}^{*}$ in Fig. 2(b)]. The top Si-rich strip represents an interlayer between two successive ribbon bundles, (b) SAED pattern of the nanoribbon $\mathrm{GG}^{*}$ where a spot diffraction pattern, assigned for the hexagonal graphite structure, is superimposed on the single crystal $4 \mathrm{H}-\mathrm{SiC}$ epilayer pattern shown in (c), and (c) SAED pattern of the single crystal $4 \mathrm{H}-\mathrm{SiC}$ epilayer.

on the single crystal pattern [Fig. 4(c)] of the $\mathrm{SiC}$ epilyer. Attributing the additional spot diffraction pattern to the hexagonal graphite structure, the direction of 0002 reflection spot of the graphite structure is found to be almost perpendicular (interplaner angle $\sim 87^{\circ}$ ) to the direction of 0002 reflection spot of the $\mathrm{SiC}$ epilayer. The interplanar distance of the (0002) graphite lattice is determined to be $0.324 \mathrm{~nm}$ based on the SAED pattern in Fig. 4(b) by considering the

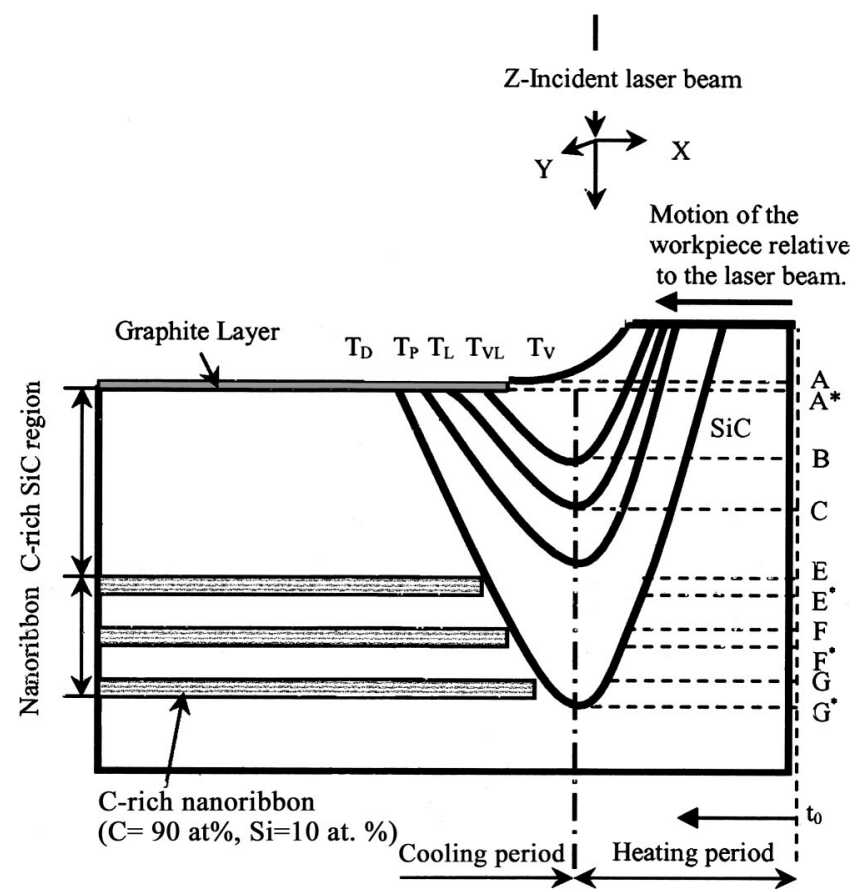

FIG. 5. Schematic representation of different isotherms in the $X Z$ plane of the laser-irradiated wafer. $T_{V}$-complete vaporization isotherm above which $\mathrm{SiC}$ exists only in the vapor phase according to the $\mathrm{Si}-\mathrm{C}$ phase diagram, $T_{V L}$-partial vaporization isotherm above which $\mathrm{Si}$ vaporizes from the liquid phase, $T_{L}-\mathrm{SiC}$ liquids temperature above which $\mathrm{SiC}$ exists only in the liquid form according to the phase diagram, $T_{P}$-peritectic decomposition isotherm above which $\mathrm{SiC}$ decomposes to form liquid $\mathrm{Si}$ that dissolves a certain amount of $\mathrm{C}$ and the rest of $\mathrm{C}$ remains as solid particles in the liquid solution phase, and $T_{D}$-significant diffusion isotherm above which the diffusion of $\mathrm{Si}$ in the solid $\mathrm{SiC}$ phase is sufficiently large to form the observed nanoribbon structure. L2 + V-liquid and vapor phases, L2-liquid phase, $\mathrm{L} 1+\mathrm{S}$-liquid $\mathrm{Si}$ with dissolved $\mathrm{C}$ and solid $\mathrm{C}$ particles, and $\mathrm{S}-$ Solid phase.

single crystal $4 \mathrm{H}-\mathrm{SiC}$ diffraction pattern of Fig. 4(c) as a reference. It should be noted that an EDS revealed incomplete graphitization $(85-90$ at. \% C, 10-15 at. \% Si) in the nanoribbons.

\section{DISCUSSION}

We propose a mechanism for the formation of the nanoribbon structure based on both thermal and mass transport phenomena occurring due to laser irradiation, and the thermodynamic interactions between $\mathrm{Si}$ and $\mathrm{C}$ as depicted by the silicon-carbon binary phase diagram. ${ }^{7}$ Several important isotherms produced in the $\mathrm{SiC}$ wafer during laser irradiation are schematically illustrated in Fig. 5. The profiles of these isotherms do not change under quasi-steady-state condition. Different physical phenomena, e.g., vaporization, peritectic decomposition, and solid-state diffusion, occur in the regions bounded by these isotherms. At any instant of time $t_{0}$, the stoichiometric composition is the same $(\mathrm{Si} / \mathrm{C}=1)$ at a set of points $\mathrm{A}$ to $\mathrm{G}^{*}$ that are in the solid $\mathrm{SiC}$ phase in front of the laser beam. As these points move past the laser beam, they intersect different isotherms at different times and spend different amounts of time in different isotherm regions. The 
points $\mathrm{A}, \mathrm{B}, \mathrm{C}, \mathrm{E}$, and $\mathrm{G}^{*}$ are so chosen that there trajectories are tangential to the deepest points of the isotherms $T_{V}$, $T_{V L}, T_{L}, T_{P}$, and $T_{D}$, respectively.

To explain the formation of the graphite sheets observed in Fig. 3, we assume that the $T_{V}$ isotherm geometrically coincides with the lowest point on the free surface of the U-shaped trench and that the measured width of the graphite layer $(3.37 \mathrm{~nm})$ is less than the distance AB. Silicon vaporizes in the region bounded by the isotherms $T_{V}$ and $T_{V L}$, and it is, therefore, lost at the top surface of this region. This leads to the formation of a C-rich layer there, which transforms into the graphite layer $\mathrm{AA}^{*}$ upon solidification during the cooling period. The loss of carbon atoms from the graphite layer to the liquid phase in the region bounded by the isotherms $T_{V L}$ and $T_{P}$ is suppressed due to the following three reasons. First, the inherent rapid solidification in laser processing traps the carbon atoms in the solid phase, preventing them from escaping to the liquid phase. Second, the difference between the atomic masses of $\mathrm{C}$ and $\mathrm{Si}$ enhances the separation of $\mathrm{C}$ and $\mathrm{Si}$ atoms and suggests that the lighter carbon atoms will float at the surface of the Si-containing liquid phase, retarding the dissolution of C. Finally, the heat of mixing is not available to the system during the cooling period when heat is removed from the system instead of being supplied.

According to the temperature distribution in Fig. 5, only the $\mathrm{SiC}$ material initially in the range $\mathrm{AB}$ experiences a selective loss of silicon due to vaporization. However, elemental analysis of the laser-generated $\mathrm{C}$-rich $\mathrm{SiC}$ phase showed that the amount of $\mathrm{Si}$ increases gradually from the points $\mathrm{A}^{*}$ to point $\mathrm{E}$. The gradation in Si concentration and the formation of $\mathrm{C}$-rich $\mathrm{SiC}$ phase may be due to the precipitation of $\mathrm{Si}$ and rapid solidification, respectively, because the species $\mathrm{SiC}, \mathrm{Si}$, and $\mathrm{C}$ spend more time at temperatures above the peritectic reaction temperature $T_{P}$ at lower depths (near the free surface of the wafer) than at the deeper regions. This is so because the region bounded by the $T_{P}$ isotherm is wider at the top than near the bottom of the isotherm. Silicon, being a heavier element than $\mathrm{C}$, will precipitate for longer periods of time at the top region than at the bottom region, resulting in increased $\mathrm{Si}$ concentration from points $\mathrm{A}^{*}$ to $\mathrm{E}$. It should also be noted that higher temperatures at the top region induce liquid Si to dissolve more $\mathrm{C}$, since the solubility, in this case, increases as the solvent temperature increases. Due to rapid solidification of this supersaturated solution, the segregation of the solute $\mathrm{C}$ is less than the partitioning of the solute under equilibrium cooling conditions. The aforementioned effects resulted in the formation of a $\mathrm{C}$-rich $\mathrm{SiC}$ solid phase in region $\mathrm{A}^{*} \mathrm{E}$.

For the part of $\mathrm{SiC}$ wafer that does not undergo peritectic decomposition, i.e., for all points below E, solid-state diffusion of $\mathrm{Si}$ from the $\mathrm{SiC}$ lattice results in both morphological and compositional variations leading to the formation of the nanoribbon region $\mathrm{EG}^{*}$. The diffusion process continues in region $\mathrm{EG}^{*}$ during the time period spent by $\mathrm{SiC}$ in the temperature range $T_{D}$ to $T_{P}$. However, the kinetics of this process is limited by the diffusion of $\mathrm{Si}$ in $\mathrm{SiC}$ at this temperature range since the diffusivity of $\mathrm{Si}$ in $\mathrm{SiC}$ is $10^{-12}$ to $10^{-10} \mathrm{~cm}^{2} / \mathrm{s}$ in the temperature range $T=2400^{\circ} \mathrm{C}$ to $T_{P}$
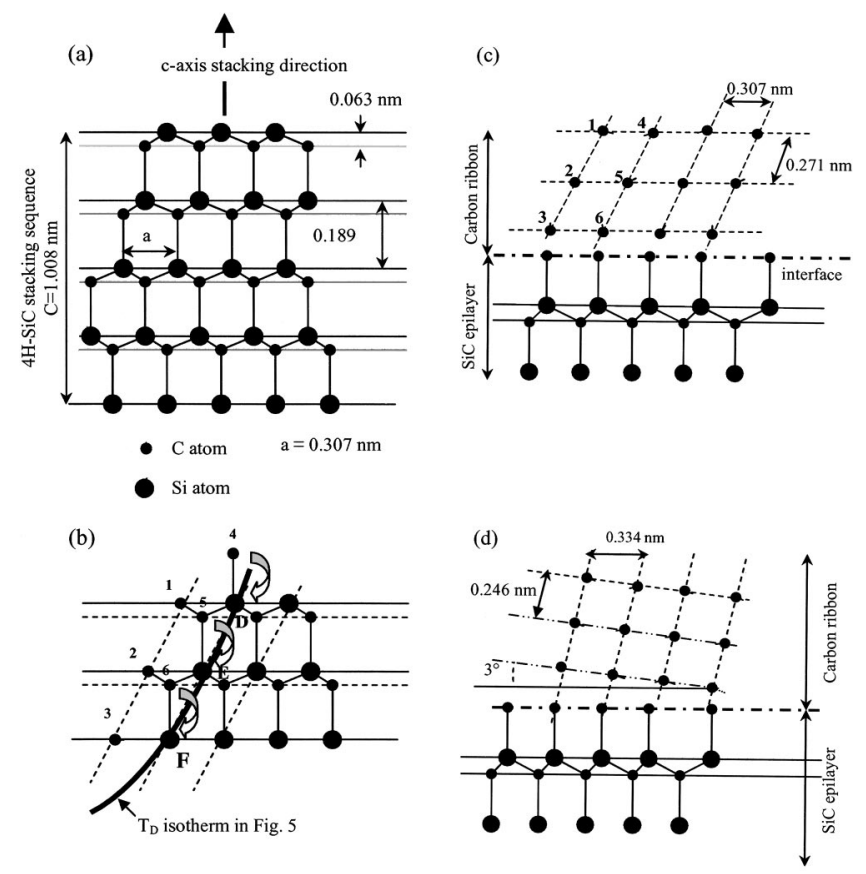

FIG. 6. Mechanism of the $\mathrm{SiC}$ decomposition leading to the formation of C-rich nanoribbon. (a) Schematic cross-section, (1120) planes, of the $4 \mathrm{H}-$ SiC lattice (see Ref. 17). (b) Collapse of the out of plane $\mathrm{C}$ atoms due to $\mathrm{Si}$ outdiffusion. (c) Arrangement of $\mathrm{C}$ atoms after step (b) causing strained ribbon-epilayer interface. (d) Rotation of the carbon ribbon in the clockwise direction around an axis normal to the plane of the paper at the ribbonepilayer interface to relieve interfacial strain.

$=2560{ }^{\circ} \mathrm{C} .{ }^{16}$ Under the present laser irradiation conditions, the laser-substrate interaction time is $0.2 \mathrm{~s}$. Based on an average diffusion coefficient of $5 \times 10^{-11} \mathrm{~cm}^{2} / \mathrm{s}$ for $\mathrm{Si}$ in $\mathrm{SiC}$, the maximum attainable $\mathrm{Si}$ diffusion distance during laser irradiation is $\sim 64 \mathrm{~nm}$.

According to this estimate, if the point $\mathrm{E}^{*}$ intersects the $T_{D}$ isotherm within approximately $0.2 \mathrm{~s}$ after point $\mathrm{E}$ did, a silicon-deficient region of thickness $64 \mathrm{~nm}$ is expected to form in the region between $\mathrm{E}$ and $\mathrm{E}^{*}$. This can explain the formation of the first of the three ribbons. The thickness of each of these ribbons is $50-60 \mathrm{~nm}$ and they are interspaced by Si-rich strips $\sim 50 \mathrm{~nm}$ thick. Now, in order for a $\mathrm{Si}$ atom in region $\mathrm{E}^{*} \mathrm{~F}$ to diffuse toward the laser-generated $\mathrm{C}$-rich phase, it has to acquire the activation energy necessary to overcome the diffusion barrier of the $\mathrm{SiC}$ lattice, traverse the two interfaces of the nanoribbon $\mathrm{EE}^{*}$, and diffuse through the C-rich nanoribbon. This process is energetically less possible than the localized diffusion and rearrangement of atoms in region $\mathrm{E}^{*} \mathrm{~F}^{*}$. Such localized phenomena lead to the formation of the nanoribbon $\mathrm{FF}^{*}$ and, similarly, $\mathrm{GG}^{*}$. Below $\mathrm{G}^{*}$, the temperature is not sufficiently high to induce $\mathrm{Si}$ outdiffusion significantly and, therefore, the epilayer maintains its original composition.

The orientation of the C-rich nanoribbon relative to the $\mathrm{SiC}$ wafer is explained in Fig. 6 based on the atomic arrangement induced in the $\mathrm{SiC}$ lattice by laser irradiation and the geometry of both the $4 \mathrm{H}-\mathrm{SiC}$ lattice and hexagonal graphite lattice. The intersheet distance of the hexagonal graphite structure is $\sim 0.334 \mathrm{~nm}$, and its $a$ and $c$ parameters are 0.246 $\mathrm{nm}$ and $0.67 \mathrm{~nm}$, respectively, while the $a$ and $c$ parameters 
of the $4 \mathrm{H}-\mathrm{SiC}$ lattice [Fig. 6(a)] are $0.307 \mathrm{~nm}$ and $1.004 \mathrm{~nm}$, respectively. ${ }^{7,15}$ The crystallographic orientation of the starting $\mathrm{SiC}$ wafer, (0001) Si face, and the observed orientation of the grown ribbons [Fig. 4(b)] suggests that the later are nucleated on the basal plane of the $\mathrm{SiC}$ lattice. If the $a$ axis of the graphite structure in the ribbons is aligned perpendicular to the $a$ axis of the $\mathrm{SiC}$ crystal, the difference between the carbon interatomic distances in the two lattices is $\Delta$ $=0.0264 \mathrm{~nm}$, i.e., $\sim 8 \%$ of the carbon spacing along the $a$ axis in the $\mathrm{SiC}$ crystal and $\sim 10 \%$ of the distance between the graphite sheets in the graphite hexagonal structure. This would cause less strain at the interface than in the case where the basal planes of the graphite hexagon are parallel to the $\mathrm{SiC}$ wafer surface with an interfacial mismatch (difference in atomic spacing) of $0.06 \mathrm{~nm}$.

However, the SAED pattern shown in Fig. 4(b) indicated that the $c$ axis in C-rich ribbons is inclined to the basal plane of the $4 \mathrm{H}-\mathrm{SiC}$ lattice at $\sim 3^{\circ}$. The following mechanism is proposed to explain this orientation relationship. When the $\mathrm{SiC}$ wafer moves with respect to the laser beam, its temperature increases gradually as it approaches the optical center of the laser beam. In a given volume of the $\mathrm{SiC}$ wafer experiencing a temperature $T$ such that $T_{P}>T>T_{D}$, Si atoms leave the $\mathrm{SiC}$ lattice due to $\mathrm{Si}$ outdiffusion. Consequently, the atomic bonds between $\mathrm{Si}$ and its four nearest-neighbor $\mathrm{C}$ atoms are broken and the out-of-plane $\mathrm{C}$ atom jumps down to occupy the Si site [Fig. 6(b)]. This process continues as the laser irradiation progresses, leading to the formation of the C-rich sheets in the nanoribbons [Fig. 6(c)].

Due to rapid heating and cooling inherent in high intensity laser irradiation, the rearranged carbon atoms in the ribbon do not reach the equilibrium graphite structure. The intersheet distance $(\sim 0.307 \mathrm{~nm})$ and the $a$ parameter $(\sim 0.271$ $\mathrm{nm})$ in the ribbon are, respectively, smaller and larger than those of the hexagonal graphite. Additionally, the carbon atoms across the epilayer-ribbon interface experience a severe lattice strain due to the atomic repulsion. A clockwise rotation [Fig. 6(d)] of the graphite sheets around an axis normal to the interface appears to be a possible mechanism for the carbon atoms in the ribbon to (i) decrease their in-plane interatomic distance ( $a$ parameter) in order to get closer to the graphite structure $(0.246 \mathrm{~nm})$, (ii) increase their intersheet distance toward the $0.334 \mathrm{~nm}$ value, and (iii) reduce the lattice strain at the interface by increasing the distance between the carbon atoms. Such a rotation can lead to the observed orientation relationship between the ribbon and $\mathrm{SiC}$ wafer.

This proposed mechanism for the nanoribbon formation is quite different from the one proposed by Van Bommel et al. ${ }^{18}$ and applied by Kushunoki et al. ${ }^{19}$ to explain the formation of graphite sheets and CNT via high-temperature decomposition of $\mathrm{SiC}$. During high-temperature decomposition of $\mathrm{SiC}$ in a vacuum, the $\mathrm{Si}$ atoms evaporate uniformly at the $\mathrm{SiC}$ surface, which leads to the collapse of the $\mathrm{C}$ atoms either by the two-layer or, for density consideration, the three-layer mechanism. The characteristics of the carbon nanostructure resulted from this uniform surface decomposition depend on the $\mathrm{SiC}$ polytype and surface polarity. ${ }^{18,19}$ In the present case, the $\mathrm{C}$-rich nanoribbons are formed inside the $\mathrm{SiC}$ wafer instead of its top surface via solid-state outdiffusion of $\mathrm{Si}$, which suggests that the growth of the nanoribbon by the laser direct-write method is independent of the polarity of the $\mathrm{SiC}$ face.

The laser direct-write doping technique discussed in this article is a nanofabrication method that is expected to significantly affect the fabrication of hybrid nanodevices involving wide band-gap materials for high-temperature and high power electronic and optoelectronic applications.

\section{CONCLUSIONS}

We have discussed a laser direct-write process to fabricate $\mathrm{C}$-rich nanoribbons in a $4 \mathrm{H}-\mathrm{SiC}$ single crystal wafer. The process relies on the compositional and morphological variations due to the mass and heat transfer patterns achieved in the wafer during laser irradiation. Three distinct regions are formed. The top graphite layer is produced due to the vaporization of $\mathrm{Si}$ and poor dissolution of $\mathrm{C}$ in $\mathrm{Si}$. The middle $\mathrm{C}$-rich $\mathrm{SiC}$ region is generated due to the precipitation of $\mathrm{Si}$ and rapid solidification of supersaturated $\mathrm{SiC}$ solution. The nanoribbon region is formed due to the solid-state diffusion. The composition, dimension, and orientation of the nanoribbons are strongly dependent on the laser processing parameters. The proposed laser process enables the fabrication of nanoribbon-SiC heterostructure directly within the $\mathrm{SiC}$ wafer and thus eliminates the need for nanostructure transferring from one medium to another. SiC-nanoribbons heterostructures are expected to be used for a wide variety of applications including interconnects for nanodevices, trench field-effect transistors, tunneling transistors, carbon nanoand microvariable resistors for devices embedded in a $\mathrm{SiC}$ wafer, and photonic band-gap arrays such as the periodic waveguide filters in the microstrip circuits.

\section{ACKNOWLEDGMENTS}

This work was supported by the AppliCote Associates and the Florida State High Technology Initiative.

\footnotetext{
${ }^{1}$ M. Kusunoki, J. Shibata, M. Rokkaku, and T. Hirayama, Jpn. J. Appl. Phys., Part 2 37, L605 (1995).

${ }^{2}$ Y. Zhang, T. Ichihashi, E. Landree, F. Nihey, and S. Iijima, Science 285, 1719 (1999).

${ }^{3}$ Y. Satio, S. Uemera, and K. Hamaguchi, Jpn. J. Appl. Phys., Part 2 37, L346 (1998).

${ }^{4}$ Q. Wang, T. Corrigan, J. Dai, R. Chang, and A. Krauss, Appl. Phys. Lett. 70, 3308 (1998).

${ }^{5}$ Q. Wang et al., Appl. Phys. Lett. 72, 2912 (1998).

${ }^{6} \mathrm{~N}$. Taguchi, US Patent, No. 3, 695, 848 (1972).

${ }^{7}$ M. E. Levinshtein, S. L. Rumayantsev, and M. S. Shur, Properties of Advanced Semiconductor Materials, 1st ed. (Wiley, New York, 2001), pp. 93-147.

${ }^{8}$ H. Dia, E. Wong, Y. Lu, S. Fan, and C. Lieber, Nature (London) 375, 769 (1995).

${ }^{9}$ J. Hu, M. Ouyang, P. Yang, and C. Lieber, Nature (London) 399, 48 (1999).

${ }^{10}$ M. Kusunoki, T. Suzuki, T. Hirayama, N. Shibata, and K. Kaneko, Appl. Phys. Lett. 77, 351 (2000).

${ }^{11}$ T. Shimizu, Y. Ishikawa, M. Kusunoki, T. Nagano, and N. Shibata, Jpn. J. Appl. Phys., Part 2 39, L605 (2000).

${ }^{12}$ N. Quick, US Patents, Nos. 5,145,741 (1992), No. 5,837,607 (1998), No. 6,025,607 (2000), and No. 6,054,375 (2000).

${ }^{13}$ I. Salama, N. Quick, and A. Kar, J. Electron. Mater. 31, 200 (2002).

${ }^{14}$ I. Salama, N. Quick, and A. Kar (unpublished).
} 
${ }^{15}$ K. Andrews, D. Dyson, and S. Keown, Interpretation of Electron Diffraction Patterns, 2nd ed. (Plenum, New York, 1971), p. 212.

${ }^{16}$ K. Ruschenschmidt, H. Brach, M. Laube, N. Stolwijk, and G. Pensl, Physica B 308, 737 (2001).

${ }^{17}$ P. Neudeck, in Very Large Scale Integrated Handbook, edited by W. K.
Chen and B. Raton (CRC and IEEE, Boca Raton, FL, 2000), pp. $6.1-6.24$.

${ }^{18}$ A. V. Bommel, J. Crombeen, and A. V. Tooren, Surf. Sci. 48, 463 (1975).

${ }^{19}$ M. Kushunoki, T. Suzuki, T. Hirayama, and N. Shibata, Physica B (to be published). 